\title{
Dielectric Breakdown of Alumina Single Crystals
}

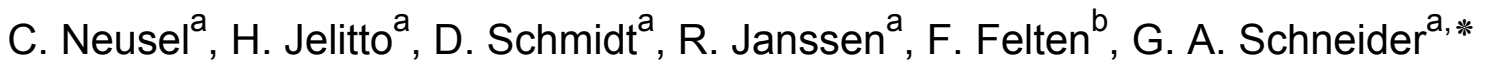

aHamburg University of Technology, Institute of Advanced Ceramics, Denickestr. 15, 21073 Hamburg, Germany

${ }^{b}$ Robert Bosch $\mathrm{GmbH}$, Corporate Sector Research and Advance Engineering, Applied Research 1 - Advanced Functional and Sintered Materials, 70049 Stuttgart, Germany

\author{
*Corresponding author: Gerold A. Schneider \\ E-Mail: g.schneider@tuhh.de
}

\begin{abstract}
The bulk breakdown behaviour of alumina single crystals with two different crystal orientations, $\{11 \overline{2} 0\}$-plane (single crystal A) and $\{0001\}$-plane (single crystal C), have been studied. Therefor plan-parallel single crystal samples were electrically loaded until dielectric breakdown was achieved. For each crystal orientation, a characteristic breakdown channel direction through the sample could be defined. In C-oriented crystals the breakdown channel originated parallel to the c-axis. For Aoriented crystals however, the breakdown channel crossed the sample in an oblique direction; the angle between crystal surface and breakdown channel was $60^{\circ}$. Here, the breakdown channel crossed the sample along an A-plane. Although the breakdown channel paths of $A$ and $C$ crystals are different, the observed breakdown strength are identical within the scatter range.
\end{abstract}

Keywords: B. Failure analysis; Optical microscopy; C. Dielectric properties; D. singlecrystal $\mathrm{Al}_{2} \mathrm{O}_{3} ; \mathrm{E}$. Insulators 


\section{Introduction}

The phenomenon of dielectric breakdown has been investigated since about 1930, by for example von Hippel ${ }^{1,2,3}$, who studied the mechanism of electric breakdown of alkali halides. Until now, the process causing breakdown in non-conducting materials is not well-understood. For example, ceramic materials which are widely used as insulation materials in industrial applications still exhibit limitations although their dielectric breakdown strength is optimized using empirical methods. The initiation of dielectric breakdown is proposed to be an electronic dominated process ${ }^{4}$ and can be described as a sudden destabilisation of charges trapped in the material ${ }^{5,6}$. Defects of the microstructure such as pores, grain boundaries, impurities, crystallographic defects and secondary phases are considered as trapping sites. Therefore, it is generally believed that the resistance against dielectric breakdown is directly connected to microstructure properties ${ }^{7,8,9,10}$. In the present study, the focus is on the dielectric breakdown behaviour of alumina single crystals. In order to eliminate microstructural effects, single crystals of two crystal orientations are used. A correlation between crystal orientation and development of the breakdown channel is proposed.

\section{Method}

\subsection{Material}

Dielectric breakdown experiments were performed on commercially available high purity alumina single crystals (Tab.1) produced by the Edge-Defined Film-Fed Growth Process $^{11}$. Cylindrical samples with a diameter of $30 \mathrm{~mm}$ and thickness of $1.5 \mathrm{~mm}$ were cut and subsequently the surfaces of the samples were epitaxial polished on both sides at CrysTec Kristalltechnologie (Berlin, Germany). The two different crystal orientations tested were A-plane and C-plane, parallel to the $\{11 \overline{2} 0\}$ and $\{0001\}$-plane (Fig. 1 ) respectively. In this report, samples with a surface parallel to the $\{11 \overline{2} 0\}$-plane will be labelled as single crystals $A$ while samples with a surface parallel to the $\{0001\}$-plane will be labelled as single crystals $C$. 
a)

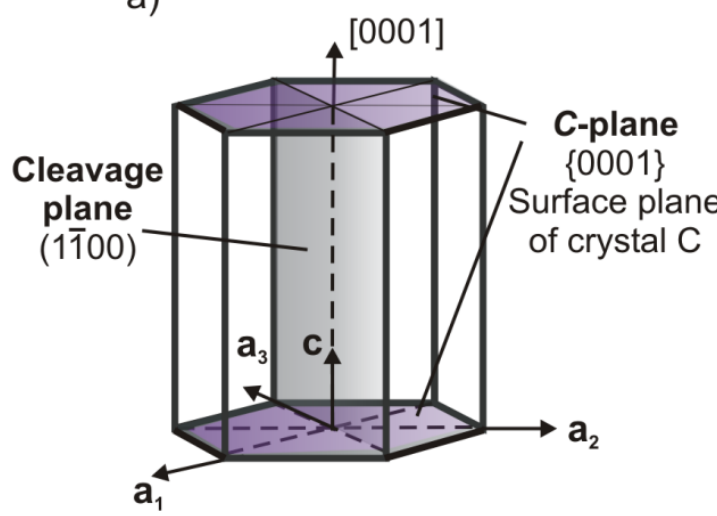

b)

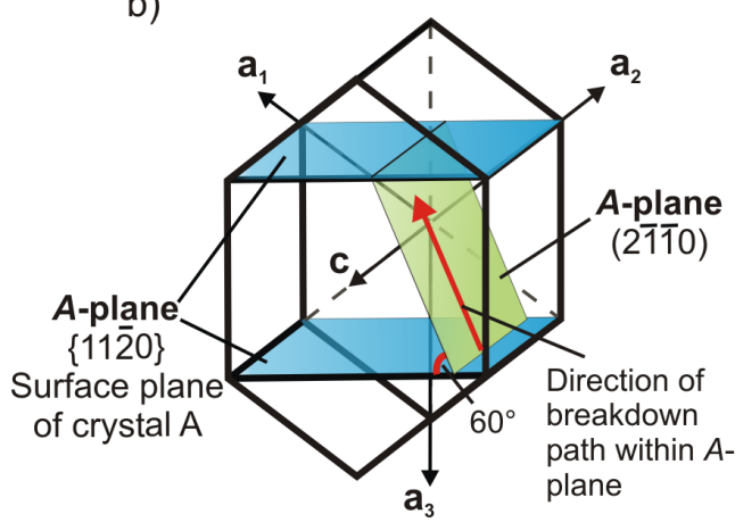

Fig. 1: Schematic picture of surface planes (a) of crystal $C$ and (b) crystal A.

Table 1: Impurity content of alumina single crystal measured by Optical Emission

Spectrometer and Mass-Spectrometer.

\begin{tabular}{cccc}
\hline \hline Element & $\begin{array}{c}\text { Content } \\
{[\mathrm{ppm}]}\end{array}$ & Element & $\begin{array}{c}\text { Content } \\
{[\mathrm{ppm}]}\end{array}$ \\
\hline $\mathrm{Si}$ & 10 & $\mathrm{~K}$ & 1 \\
$\mathrm{~S}$ & 4 & $\mathrm{Ca}$ & 1 \\
$\mathrm{Fe}$ & 2 & $\mathrm{Ti}, \mathrm{Y}$ & $<1$ \\
$\mathrm{Na}$ & 1 & $\mathrm{Zr}, \mathrm{Cr}$ & $<1$ \\
\hline \hline
\end{tabular}

Cata from CrysTec Kristalltechnologie, Berlin.

\subsection{Breakdown test}

The breakdown tests were performed in silicone oil at room temperature using rectified ac voltage. For each crystal orientation, 11 samples were tested. The high voltage electrode of the measuring cell was a brass-made pin electrode with a rounded tip, enclosed with an unplasticized PVC cylinder to avoid flashover behaviour. The ground-electrode made of stainless steel has a flat surface area for contact with the sample and a rounded edge, similar to a Rogowski profile. To ensure a good electrical connection between sample and electrodes, a circular shaped layer of conductive silver with a diameter of $10 \mathrm{~mm}$ was deposited on the sample surface. The samples with conductive silver electrodes were heated in a drying furnace at $100^{\circ} \mathrm{C}$ for $5 \mathrm{~h}$ before testing to ensure the evaporation of solvents. 
For breakdown, a voltage pulse with a frequency of $50 \mathrm{~Hz}$ generated by a function generator (Agilent $33220 \mathrm{~A}$ ) was amplified from low voltage to high voltage via vacuum tubes, inductors and a transformer coil.

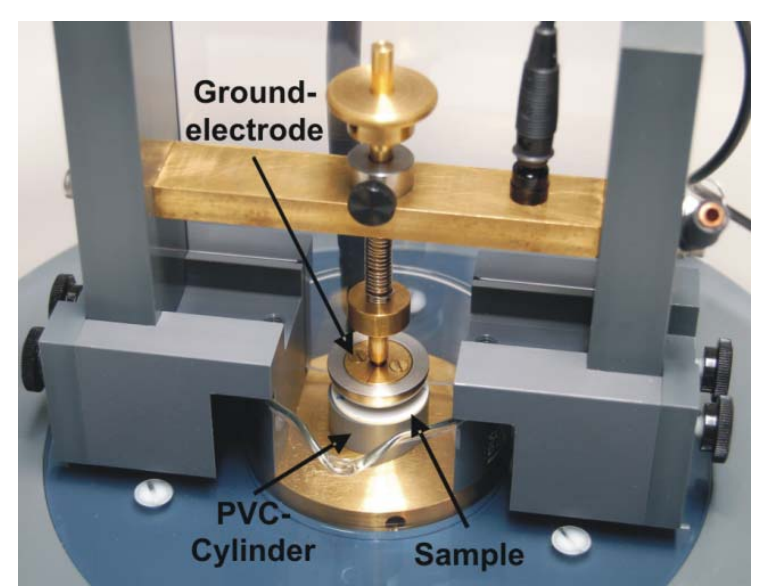

Fig. 2: Measuring Cell: the high voltage pin-electrode is enclosed by a PVC cylinder to prevent flashover behaviour between high-voltage electrode and ground-electrode.

For the breakdown tests, the samples were placed between the electrodes and immersed in silicon oil. The high voltage signal was increased by $0.2 \mathrm{kV} / \mathrm{s}$ until the sample underwent breakdown, characterised by a collapsing voltage signal detected with an oscilloscope. In most cases, the dielectric breakdown occurred under emission of light and noise.

\section{Results}

\subsection{Optical Observation of Breakdown Channel}

Fig. 3a shows a light microscopy image of the top view of a single crystal $C$ surface ( $\{0001\}$-plane), exhibiting the opening of the breakdown channel beside the conductive silver electrode. The breakdown channel is the centre of regularly, starshaped arranged cracks. The angle between adjacent cracks is $60^{\circ}$ which indicates cleavage at $\{10 \overline{1} 0\}$-planes of the hexagonal crystal structure. 
(a)

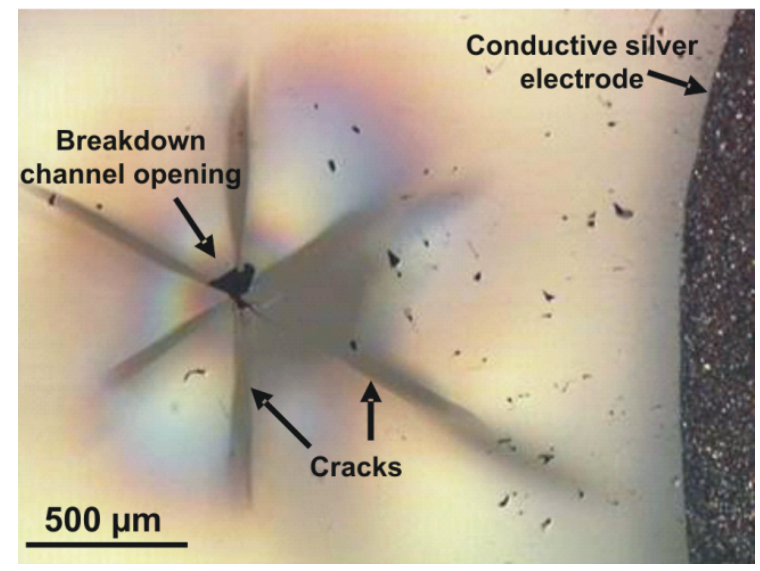

(b)

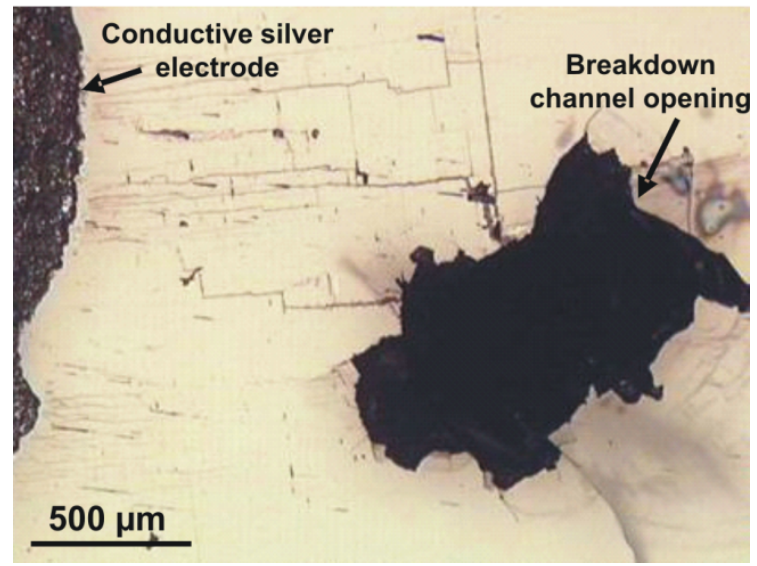

Fig. 3: Optical micrograph with polarisation filter of a characteristic breakdown channel opening (a) of single crystal $C$ and $(b)$ of single crystal $A$.

A polarising filter was used to visualize the internal stresses between the cleavage planes in Fig. 3a which surround the breakdown channel along the whole length. In Fig. $3 b$ the light microscopy image of the top view of a single crystal A surface ( $\{11 \overline{2} 0\}$-plane) including the opening of the breakdown channel besides the conductive silver electrode is shown. In contrast to single crystal $\mathrm{C}$, the channel opening of single crystal $A$ is associated with a big crater and irregularly arranged cracks. In all cases the breakdown channel originates besides the conductive silver electrode.

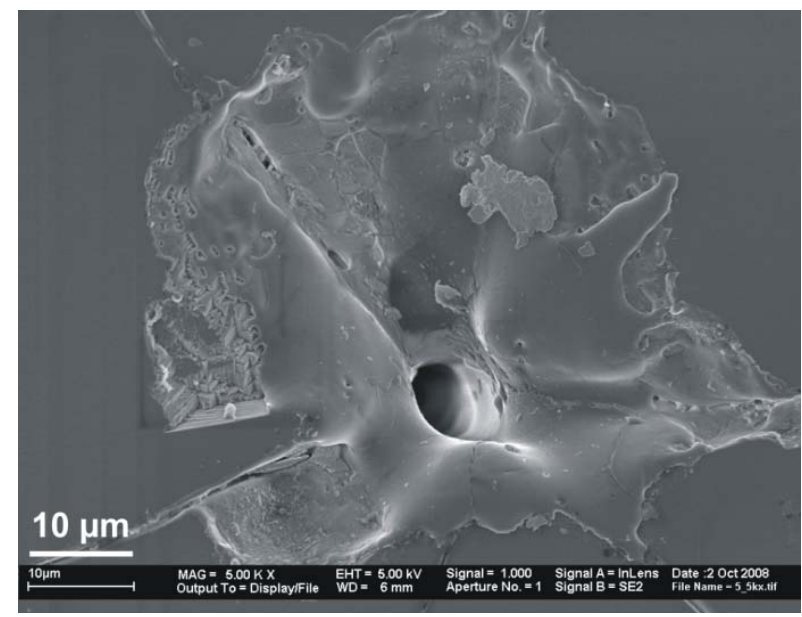

Fig. 4: SEM-picture of channel opening in single crystal C surrounded by molten material.

Molten material encloses the breakdown channel opening of single crystal C (Fig. 4) which proves the presence of high temperatures during or directly after the breakdown event. Presumably a certain amount of alumina also vaporized during the 
breakdown process; this happens suddenly, leading to compressive stresses inside the breakdown channel and tangential tensile stresses in the channel wall.

Besides the breakdown channel, typical triangular shaped deteriorations and surface grooves arranged in $120^{\circ}$ angle are found at the high voltage side of the single crystal C surface (Fig. 5a).

(a)

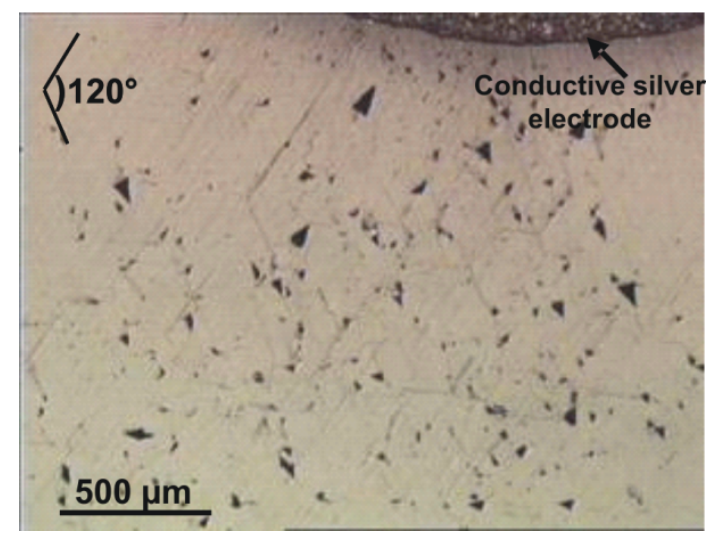

(b)

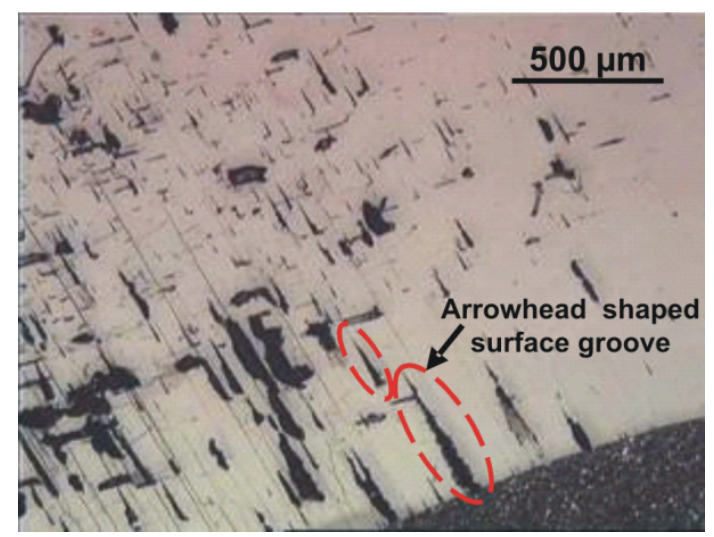

Fig 5: Typical surface deterioration due to partial discharges of (a) single crystal $C$ and (b) single crystal A.

This surface damage surrounding the conductive silver electrode is probably produced by partial discharges emitted from field enhancement at the conductive silver edge. The samples could withstand a certain amount of these partial discharges before breakdown. Additionally, the observation that the origin of the breakdown channel is not necessarily placed in an area of high surface damage indicates that these partial discharges did not immediately result in dielectric bulk breakdown.

The surface damage of single crystal $A$ before breakdown is characterised by arrowhead shaped craters (circled in Fig. $5 \mathrm{~b}$ ); $90^{\circ}$ angles are found between surface grooves. To follow the path of the breakdown channel through the single crystals $A$ and $C$, the cross-section areas perpendicular to the crystal surfaces were prepared. Fig. 6a shows the path of the breakdown channel through single crystal $\mathrm{C}$. The breakdown channel is aligned perpendicular to the sample surface, along the crystallographic c-axis of the hexagonal structure. Beside the breakdown channel, one of the $\{10 \overline{1} 0\}$-cleavage planes can be seen (dark shaded area). 
(a)

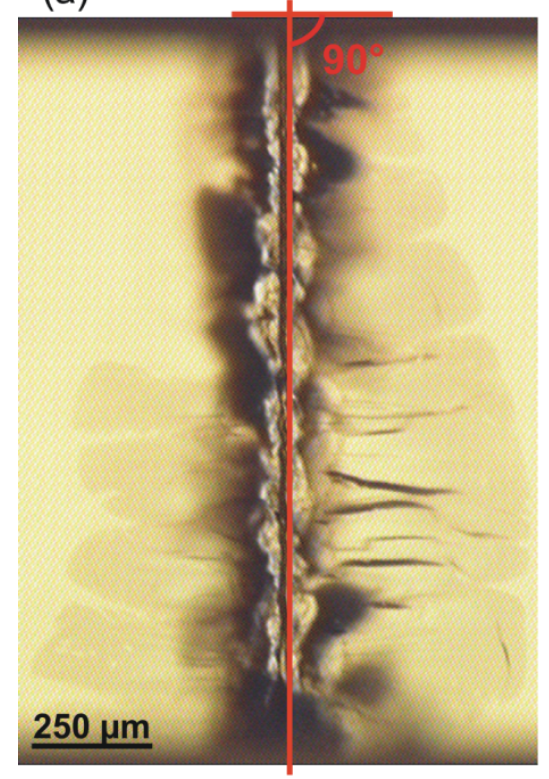

(b)

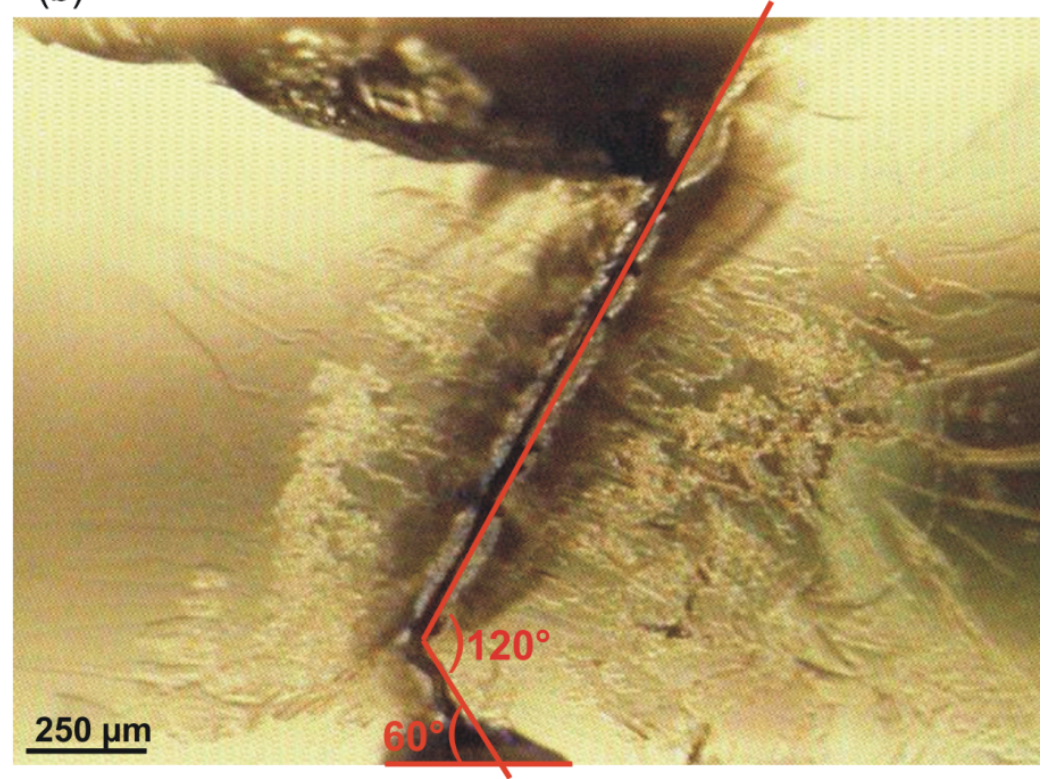

Fig. 6: Path of breakdown channel through (a) single crystal $C$ and (b) single crystal A.

In contrast to the breakdown channel profile of single crystal $\mathrm{C}$, the breakdown channel of single crystal A (Fig. 6b) is deflected by an angle of $60^{\circ}$. The breakdown channel path of single crystal A proceeds along an A-plane which is oriented with an angle of $60^{\circ}$ to the surface plane. In some single crystal A samples the breakdown channel kinked $120^{\circ}$ to another A-plane. Along the breakdown channel $\{11 \overline{2} 0\}$ cleavage planes are observed. But especially when the breakdown channel changes the direction by $120^{\circ}$, the created cleavage planes belong to a different plane family. Cleavage of the basal $\{0001\}$-plane, although crossed by the breakdown channel, is unlikely due to the high fracture surface energy of this plane ${ }^{12,13}$.

In Fig. 7, a schematic picture of the hexagonal basal plane of $\mathrm{Al}_{2} \mathrm{O}_{3}$ with $2 / 3$ filled octahedral sites' including the a-axis is given. The surface plane of single crystal A is orthogonal to the paper plane, and indicated by a blue line. The directions of the breakdown channel in a $60^{\circ}$ angle to the surface plane are indicated by red arrows. In this direction the density of atoms is lower due to the combination of low oxygen atom density and high octahedral vacancy density compared to any other direction. 


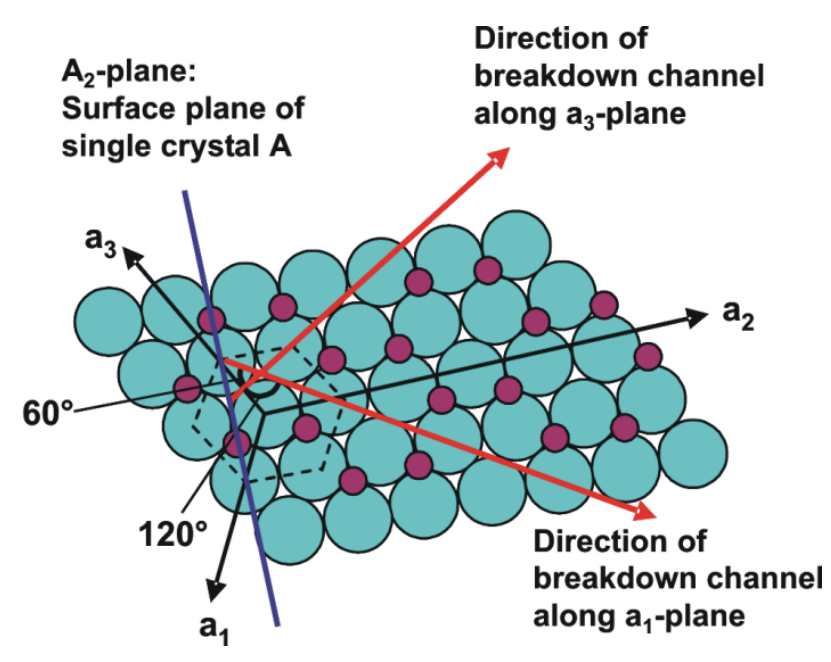

Fig. 7: Schematic picture of basal plane of $\mathrm{Al}_{2} \mathrm{O}_{3}$; oxygen atoms turquoise coloured, alumina atoms bordeaux coloured. The surface plane of the single crystal A (blue line) is normal to the paper plane, as well as the c-axis. The red arrows indicate possible directions of the breakdown at $60^{\circ}$ to the surface plane.

\subsection{Breakdown Strength}

The mean breakdown voltage $V_{b}$ and corresponding critical electric field or breakdown strength $E_{b}$ for single crystal $A$ and single crystal $C$ are identical within the standard deviation (Tab. 2). The breakdown strength $E_{b}$ is defined as breakdown voltage $V_{b}$ per sample thickness $t_{0}$. The calculation does not take into account the tilted pathway of the breakdown channel in crystal $A$. If the breakdown strength $E_{b}$ is calculated considering the tilted path way, the breakdown strength $E_{b}$ of single crystal A decreases by a factor of $\cos \left(30^{\circ}\right)=0,866$, due to the increased path length. Taking the tilted path way into account the resulting breakdown strength $E_{\mathrm{b}}$ is $(20.8 \pm 1.6) \mathrm{kV} / \mathrm{mm}$ for single crystal A. Nevertheless compared to single crystal C, no significant difference in breakdown strength can be found due to the overlapping of the standard deviations.

\section{Discussion}

The optical investigation of the breakdown channel in alumina single crystals showed that defined directions are preferred as breakdown path. In crystal C (Fig. 6a), the 
breakdown channel is parallel to the c-axis. $\{10 \overline{10}\}$-cleavage planes are developed in a star-shape manner, during the breakdown process. Furthermore molten debris around the breakdown channel opening (Fig. 4) indicates a "thermal character" of the breakdown event. Presumably, the heat generation during breakdown leads to a rapid volume increase of molten material inside the channel. This effect is enhanced by an explosion event linked to the vaporization of some alumina material. Both effects induce circumferential tensile stresses in the channel wall, leading to the formation of radial cracks along distinct cleavage planes; here along a family of $\{10 \overline{1} 0\}$-planes which intersects along the c-axis. In contrast to crystal $C$, the breakdown channel of crystal A did not cross the sample along the shortest path, perpendicular through the surface. Instead, the breakdown channel in crystal A is developed along a $\{11 \overline{2} 0\}$-cleavage plane indicated by a $60^{\circ}$ angle between surface plane and breakdown channel.

Similar cleavage behaviour during electric breakdown for an alumina single crystal has already been reported by Malec et al. ${ }^{14}$ but without any information concerning the crystal orientation.

Fracture mechanics experiments on alumina single crystals confirm that $\{11 \overline{2} 0\}$ - and $\{10 \overline{1} 2\}$-planes are favoured crack propagation directions ${ }^{13}$. The cleavage toughness of these planes is therefore low compared to a $\{0001\}$-plane. As a consequence, the formation of a $\{11 \overline{2} 0\}$-cleavage plane in single crystals $A$ is more distinct compared to the formation of a $\{0001\}$-cleavage plane. However, the question still arises why the channel is not formed along the shortest way, perpendicular through the sample surface along a $\{10 \overline{1} 2\}$-cleavage plane, which is an observed cleavage plane in alumina single crystals. Von Hippel ${ }^{1,2,3}$ studied the breakdown behaviour of alkali halides like $\mathrm{NaCl}$ and reported that for low applied electric fields, the dielectric breakdown favours the crystal direction with lowest potential barrier between similar lattice atoms. For high electric fields, the direction which allows the highest electron mobility will be favoured by the breakdown path. This direction is in most cases equal to the direction of lowest atom density. In Fig. 7, the schematic of the basal plane of the hexagonal structure is shown, including the channel path along an A-plane marked by red arrows. Along these directions, the oxygen atom density is lower compared with any other direction. Additionally, these distinct directions show the 
highest octahedral vacancy density; these are the directions of lowest atom density. Presumably, this combination of low oxygen atom density and high octahedral vacancy density causes the oblique breakdown path in the single crystal A.

In some samples a kinking of the channel profile by an angle of $120^{\circ}$ was observed. Here, the channel changed the direction from one A-plane to another A-plane. This behaviour could be caused by the position of the breakdown channel in relation to the conductive silver electrodes, which are applied onto the sample surface. In contrast to straight electric field lines directly below the silver electrode, the field lines are bent adjacent to the silver electrode. Thus, if the breakdown event is assumed to be an electronic process, the electrons will follow the electric field lines.

According to von Hippel ${ }^{3}$ surface discharges also follow preferred crystallographic directions, similar to the breakdown path of the bulk material. In Fig. 5a typical shaped surface grooves of single crystal $C$ enclosing a $120^{\circ}$ angle are shown. In case of crystal $C$ the basal plane is the surface plane, therefore the schematic picture of Fig. 7 also shows the top view onto crystal C. The directions of lowest atom density, namely the direction of low oxygen atom density in combination with high octahedral vacancy density are marked by red arrows. These directions of low atom density are arranged in $120^{\circ}$ angle to each other, similar to the surface grooves of crystal C. If the same are true for the surface deteriorations of crystal A (Fig. 5b), the arrowhead shaped surface grooves will be aligned along the c-axis.

The reported results of breakdown strength for $1.5 \mathrm{~mm}$ thick single crystals $A$ and single crystals $\mathrm{C}$ are $(24.0 \pm 1.8) \mathrm{kV} / \mathrm{mm}$ and $(23.7 \pm 1.4) \mathrm{kV} / \mathrm{mm}$, respectively.

\section{Conclusion}

Breakdown channel profiles as well as the surface deteriorations are characteristics for crystal A and crystal C. In crystal C, the breakdown channel is created parallel to the c-axis, enclosed by $\{10 \overline{10}\}$-cleavage planes which probably originate due to thermal stresses around the breakdown channel. The cleavage planes are arranged in $60^{\circ}$ angle to each other. In contrast to crystal $C$ the breakdown channel of crystal $A$ passes the sample within a $\{11 \overline{2} 0\}$-plane (A-plane), along the direction of the lowest 
atom density. This direction is favourable due to the low potential barrier when compared to other directions. The cleavage plane created by the breakdown channel in crystal $A$ is a $\{11 \overline{2} 0\}$-plane. Some of the crystals $A$ showed a kinking of the breakdown channel by $120^{\circ}$, here the path changed from one A-plane to another Aplane. This change in breakdown channel direction is also connected by a change of cleavage plane type that is created. It is assumed that the channel bending is caused by the position of the breakdown channel adjacent to the conductive silver electrode, where the electric field lines are bent.

Surface deteriorations of crystal $\mathrm{C}$ such as material chipping showed a triangular shape. The surface grooves enclose a $120^{\circ}$ angle, presumable they originate along the paths of lowest atom density which are arranged in $120^{\circ}$ to each other. The surface deteriorations of crystal $A$ showed a more rectangular form; probably their distinct orientation is the c-direction of the crystal. The breakdown strength for single crystal $A$ and single crystal $C$ are measured as $(24.0 \pm 1.8) \mathrm{kV} / \mathrm{mm}$ and $(23.7 \pm 1.4) \mathrm{kV} / \mathrm{mm}$, respectively.

\section{References}

1. von Hippel A. Der Mechanismus des „elektrischen“ Durchschlags in festen Isolatoren I. Zeitschrift für Physik 1931;67:707 - 724

2. von Hippel A. Der Mechanismus des „elektrischen“ Durchschlags in festen Isolatoren II. Zeitschrift für Physik 1931;68:309 - 324

3. von Hippel A. Der Mechanismus des „elektrischen“ Durchschlags in festen Isolatoren III. Zeitschrift für Physik 1932;75:145 - 170

4. Owate I.O. and Freer R. AC breakdown characteristic of ceramic materials. J App Phys 1992;72:2418 - 2422

5. Touzin M., Goeuriot D., Guerret-Piécourt C., Juvé D., Fitting H.-J. Alumina based ceramics for high-voltage insulation. J Eur Ceram Soc 2010;30:805 - 817

6. Touzin M., Goeuriot D., Fitting H.-J., Guerret-Piécourt C., Juvé D., Tréheux D. Relationship between dielectric breakdown resistance and charge transport in alumina materials - Effects of microstructure. J Eur Ceram Soc 2007;27:1193 1197 
7. Liebault J., Vallayer D., Goeuriot D., Treheux D., Thevenot F. How the trapping can explain the dielectric breakdown performance of alumina ceramics.

J Eur Ceram Soc 2001;21:389 - 397

8. Si Ahmed A., Kansy J., Zarbout K., Moya G., Liebault J., Goeuriot D. Microstructural origin of the dielectric breakdown strength in alumina: A study by positron lifetime spectroscopy. J Eur Ceram Soc 2005;25:2813 - 2816

9. Zarbout K., Moya G., Si Ahmed A., Damamme G., Kallel A. Study of discharge after electron irradiation in sapphires and polycrystalline alumina. $\mathrm{J}$ App Phys 2010;108:094109-1 - 094109-8

10. Haddour L., Mesrati N., Goeuriot D., Thréheux D. Relationships between microstructure and dielectric properties of different alumina materials. $J$ Eur Ceram Soc 2009;29:2747 - 2756

11. LaBelle Jr. H.E., Mlavsky A.L. Growth of controlled profile crystals from the melt: Part I - Sapphire filaments. M. R. Bulletin 1971;6:571 - 579

12. Wiederhorn S. M. Fracture of Sapphire. J Am Ceram Soc 1969;52(9):485 - 491

13. Iwasa M. and Bradt R. C. Fracture toughness of Single-Crystal Alumina. In: Kingery W.D., Advances of Ceramics Volume 10, Structure and properties of $\mathrm{MgO}$ and $\mathrm{Al}_{2} \mathrm{O}_{3}$ Ceramics 1984, p. $767-779$

14. Malec D., Bley V., Talbi F., Lalam F. Contribution to the understanding of the relationship between mechanical and dielectric strength of Alumina. J Eur Ceram Soc 2010;30:3117 - 3123 